\title{
Radioelectric asymmetric brain stimulation and lingual apex repositioning in patients with atypical deglutition
}

This article was published in the following Dove Press journal:

Journal of Multidisciplinary Healthcare

20 June 2011

Number of times this article has been viewed

\section{Alessandro Castagna' \\ Salvatore Rinaldi ${ }^{1,2}$ \\ Vania Fontani' \\ Piero Mannu'}

'Rinaldi-Fontani Institute, ${ }^{2}$ School of Occupational Medicine, University of Florence, Florence, Italy
Correspondence: Salvatore Rinaldi Rinaldi-Fontani Institute, Viale Belfiore 43, 50144 Florence, Italy

Tel +39055290307

Fax +390 55290399

Email srinaldi@irf.it
Background: Atypical deglutition is exacerbated by stress and anxiety. Several therapeutic approaches have been employed to treat stress and anxiety disorders, ranging from typical psychopharmacological strategies to novel physical protocols, such as transcranial magnetic stimulation and radioelectric asymmetric conveyor (REAC) stimulation. The purpose of the present study was to test the efficacy of REAC brain stimulation in atypical deglutition.

Methods: The position of the lingual apex (Payne method), pattern of free deglutition, and subjective and objective impression of deglutition were evaluated in 128 outpatients suffering from atypical deglutition. Deglutition testing consisted of an operator holding down the lower lip, hence counteracting the strength exerted by the orbicularis muscle. All subjects were treated using two REAC brain stimulation protocols. Patients were assessed before treatment, immediately after treatment, and three months following the last cycle of REAC therapy.

Results: REAC stimulation led to an improvement in positioning of the lingual apex and a significant decrease of muscle involvement in all patients immediately after REAC treatment, and the improvement was maintained at three-month follow-up.

Conclusion: In the present study, the REAC therapeutic protocols led to normalization in lingual apex positioning and significant improvement in swallowing in all participants suffering from atypical deglutition.

Keywords: atypical deglutition, stress, anxiety, radioelectric asymmetric brain stimulation

\section{Introduction}

Atypical deglutition ${ }^{1-11}$ is a pathological phenomenon affecting an increasing number of people in the general population. ${ }^{12} \mathrm{AD}$ is characterized by the persistence in adults of swallowing typical in infants. Several studies have investigated the etiology ${ }^{13-20}$ and treatment ${ }^{21-24}$ of the condition. Despite few relevant references in the literature, ${ }^{25,26}$ it is well accepted that atypical deglutition is linked to several stress conditions and, as such, treatment has recently focused on stress and anxiety reduction. The purpose of the present study was to evaluate the efficacy of treatment using brain stimulation with a radioelectric asymmetric conveyor (REAC), ${ }^{27,28}$ specifically developed for progressive and long-term neuropsychophysical optimization of global brain function. It was hypothesized that the objective and subjective clinical picture of patients suffering from atypical deglutition may be significantly improved by reduction of stress using REAC brain stimulation.

\section{Materials and methods}

This naturalistic study ${ }^{29}$ was conducted in 128 patients suffering from atypical deglutition (68 males and 60 females) aged 9-27 years. Participants were referred by their dentists to the Rinaldi-Fontani Institute in Florence, Italy, which has 
specialized in the diagnosis and treatment of a broad range of stress-related disorders for over 20 years. Patients were dissatisfied with the results of their ongoing orthodontic treatment and had specifically requested nonorthodontic or nonpharmacological treatment. Patients were subjected to a preliminary clinical examination, including assessment of mimic muscle involvement in deglutition, lingual apex position by distribution of fluorescein in the mouth using the Payne method, ${ }^{30}$ and an objective assessment by the physician of deglutition ability by pinching and lowering of the patient's lower lip to counteract the force exerted by the orbicularis muscle. After a baseline assessment, the first REAC brain stimulation protocol, ie, neuropostural optimization, ${ }^{31}$ was administered. This protocol, consisting of a single $500 \mathrm{msec}$ pulse in a specific area of the ear located at the top of the lower third of the scapha, was

Table I Treated groups and results
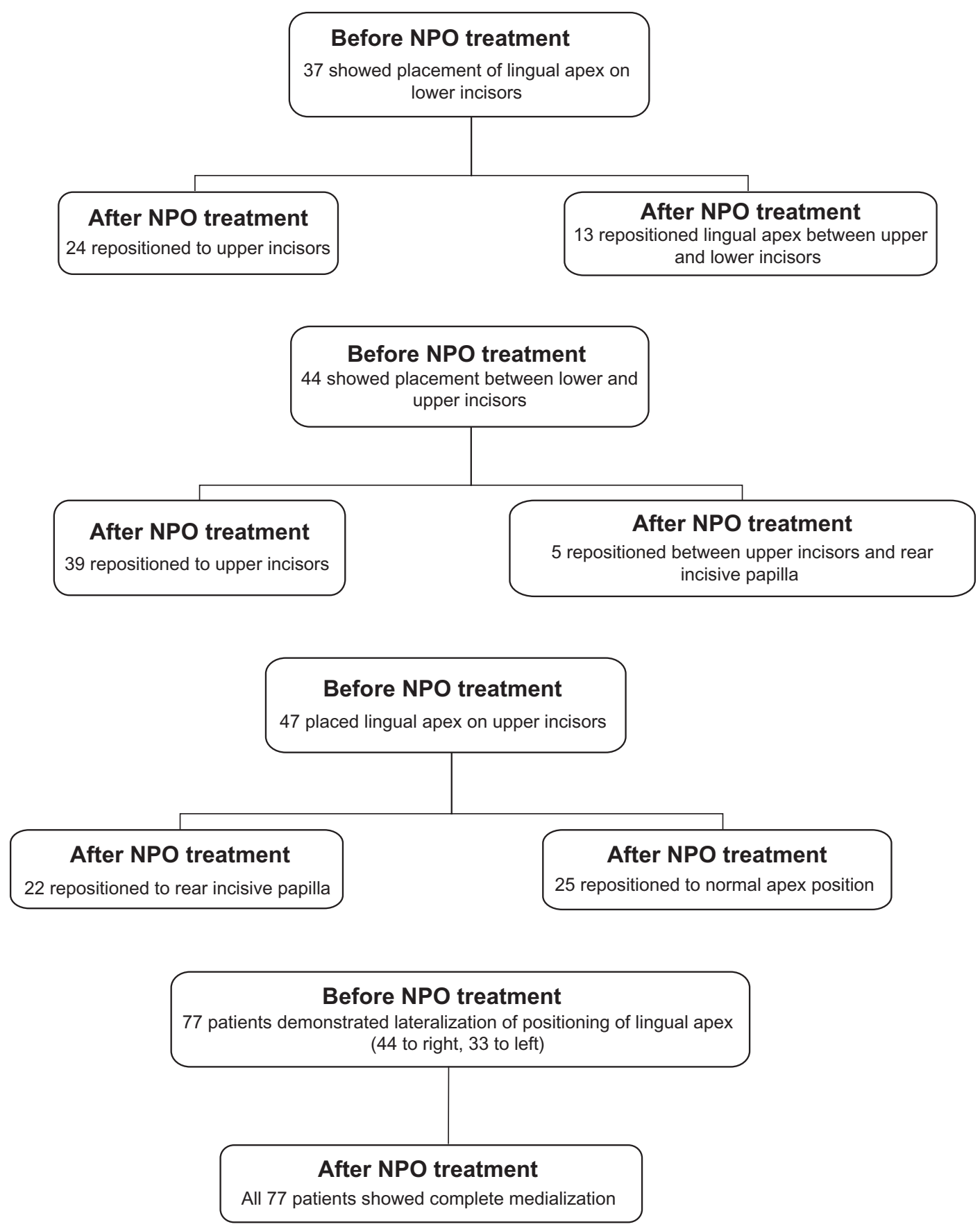

Abbreviation: NPO, neuropostural optimization. 
specifically designed for reorganization of neuromuscular control. Clinical evaluation was repeated 10 minutes following REAC

During the second phase of the study, patients received the REAC treatment, ie, neuropsychophysical optimization, which is specifically designed for stress-related disorders. ${ }^{32-37}$ Neuropsychophysical optimization consists of a sequence of seven $500 \mathrm{msec}$ pulses on seven reflex auricular points (vegetative, kidney, stomach, heart, occipital, hypothalamic, and prefrontal cortex points) in an attempt to reproduce the physiological response to stress. The neuropsychophysical optimization treatment cycle consisted of 18 therapy sessions, administered on alternate days. Clinical evaluations were repeated at the end of treatment and at a three-month follow-up visit. All procedures were carried out in accordance with the ethical principles for medical research involving human subjects laid down by the World Medical Association Declaration of Helsinki. Each patient or parent/guardian signed their written informed consent prior to entry into the study.

\section{REAC device}

The REAC is a medical biostimulation device based on innovative technology. ${ }^{27,28}$ The frequency setting of the device (typically $2.4,5.8$, or $10.5 \mathrm{gHz}$ ) is selected by the operator for each specific protocol. Our protocol for this study used a frequency of $10.5 \mathrm{gHz}$, with a specific absorption rate of $7 \mu \mathrm{W} / \mathrm{kg}$. The REAC pulse consisted of seven radiofrequency bursts of $500 \mathrm{msec}$ each, applied by touching the metallic tip of the REAC probe to the ear pavilion according to the neuropostural optimization and neuropsychophysical optimization protocols. REAC stimulation has been shown to be an effective treatment for stress-related disorders, depression, and anxiety. ${ }^{32-37}$ The treatments are painless, noninvasive, and without adverse effects.

\section{Results}

At the baseline assessment, all patients showed varying degrees of muscle involvement in deglutition, especially with regard to the orbicularis muscle of the lips and the chin muscle. None of the patients examined could swallow when the operator pinched and pulled down the lower lip. All patients breathed almost exclusively through their mouths. Of the 128 patients who underwent the procedure, 37 showed placement of the lingual apex on the lower incisors, 44 showed placement between the lower and upper incisors, and 47 showed placement on the upper incisors during swallowing (Table 1). Furthermore, 77 patients demonstrated lateralization of positioning of the lingual apex, with 44 to the right and 33 to the left. After neuropostural optimization, there was a significant decrease in involvement of the mimic muscles during swallowing in all patients, both with regard to subjective perception and objective evaluation. All patients declared that swallowing was easier.

After neuropostural optimization, 24 of the 37 patients who initially placed the lingual apex on the lower incisors then repositioned to the upper incisors, while the other 13 repositioned the lingual apex from the upper to the lower incisors. Of the 44 patients who placed the lingual apex between the upper and lower incisors at baseline, 39 repositioned to the upper incisors and five repositioned between the upper incisors and the rear incisive papilla. Of the 47 patients who initially placed the lingual apex on the upper incisors, 22 repositioned to the rear incisive papilla (Table 1 and Figure 1) and 25 to the
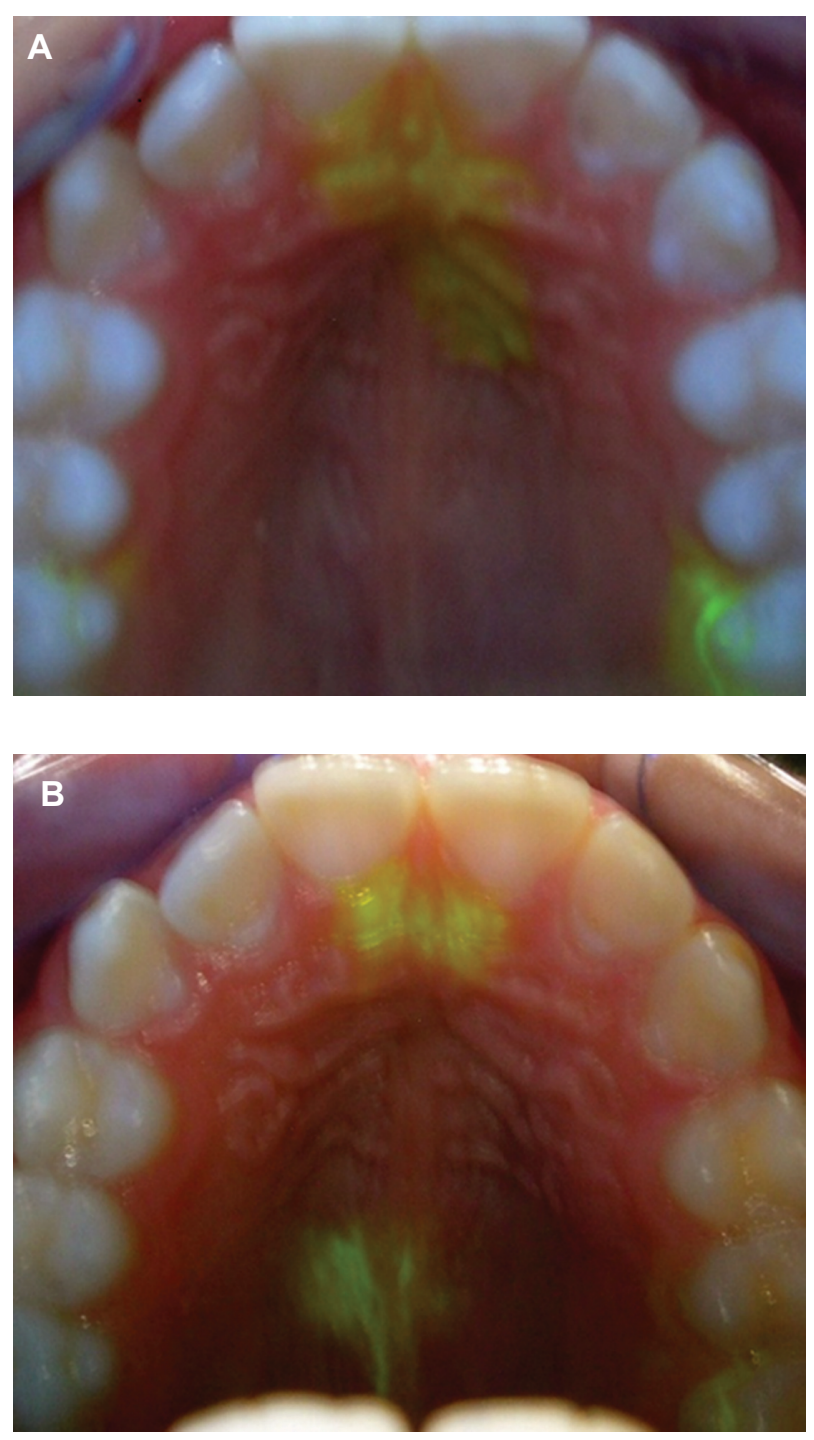

Figure I (A) Lingual apex positioned at upper incisors and (B) lingual apex repositioned to the rear incisive papilla. 

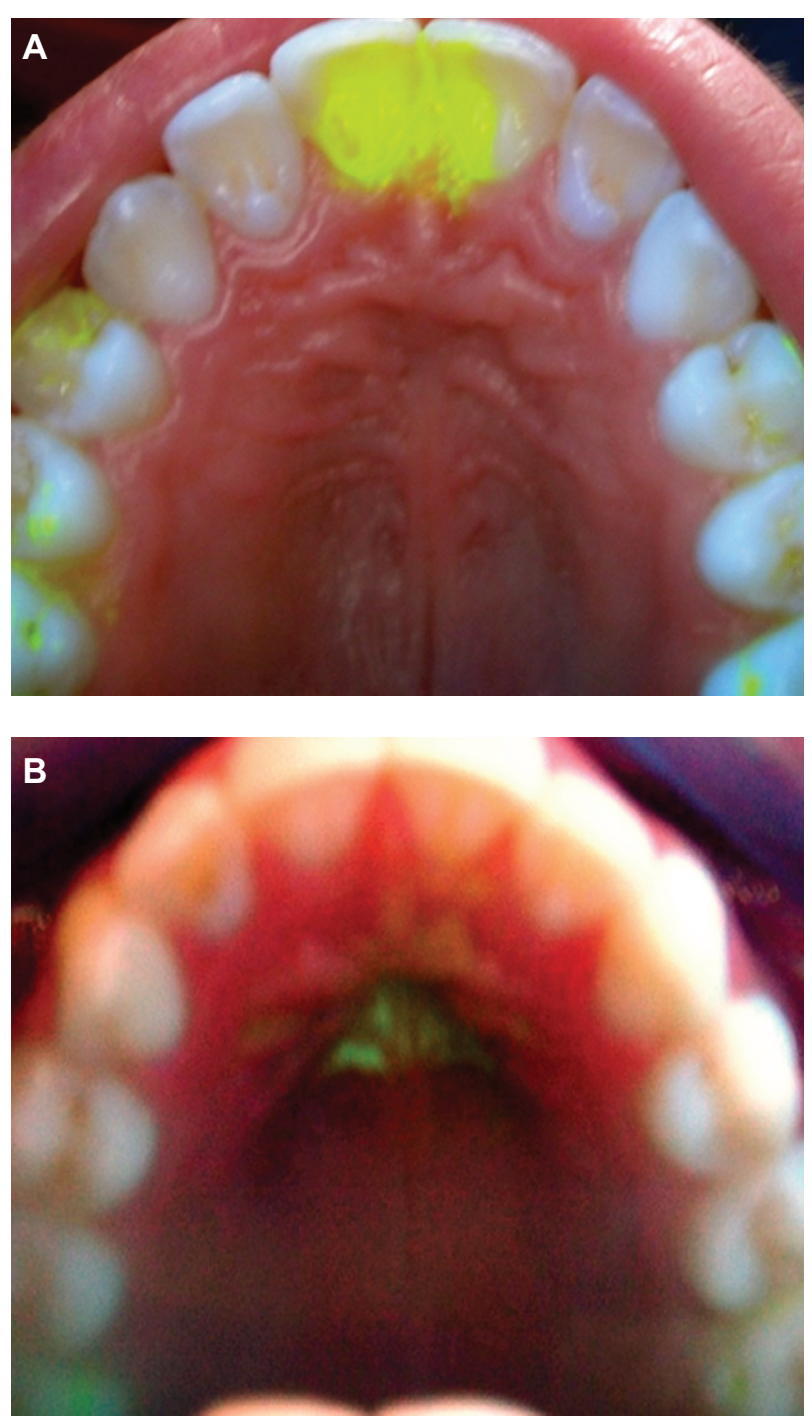

Figure 2 (A) Lingual apex positioned at the upper incisors and (B) repositioning of the lingual apex to the normal lingual apex position.

normal apex position (Table 1 and Figure 2). All 77 patients with baseline lateralization of the lingual apex showed complete medialization with treatment (Figure 3). In addition, the previous atypical propulsive swallowing pattern normalized in 20 patients. All patients swallowed more easily when the physician pinched and lowered the lower lip. Lastly, breathing was improved in all patients. At three-month follow-up, lingual apex repositioning remained stable, with further slight improvement in the majority of patients.

\section{Conclusion}

In the present study, the REAC therapeutic protocols (neuropostural optimization and neuropsychophysical optimization) led to normalization in lingual apex positioning and significant improvement in swallowing in all participants suffering from atypical deglutition. Global clinical amelioration
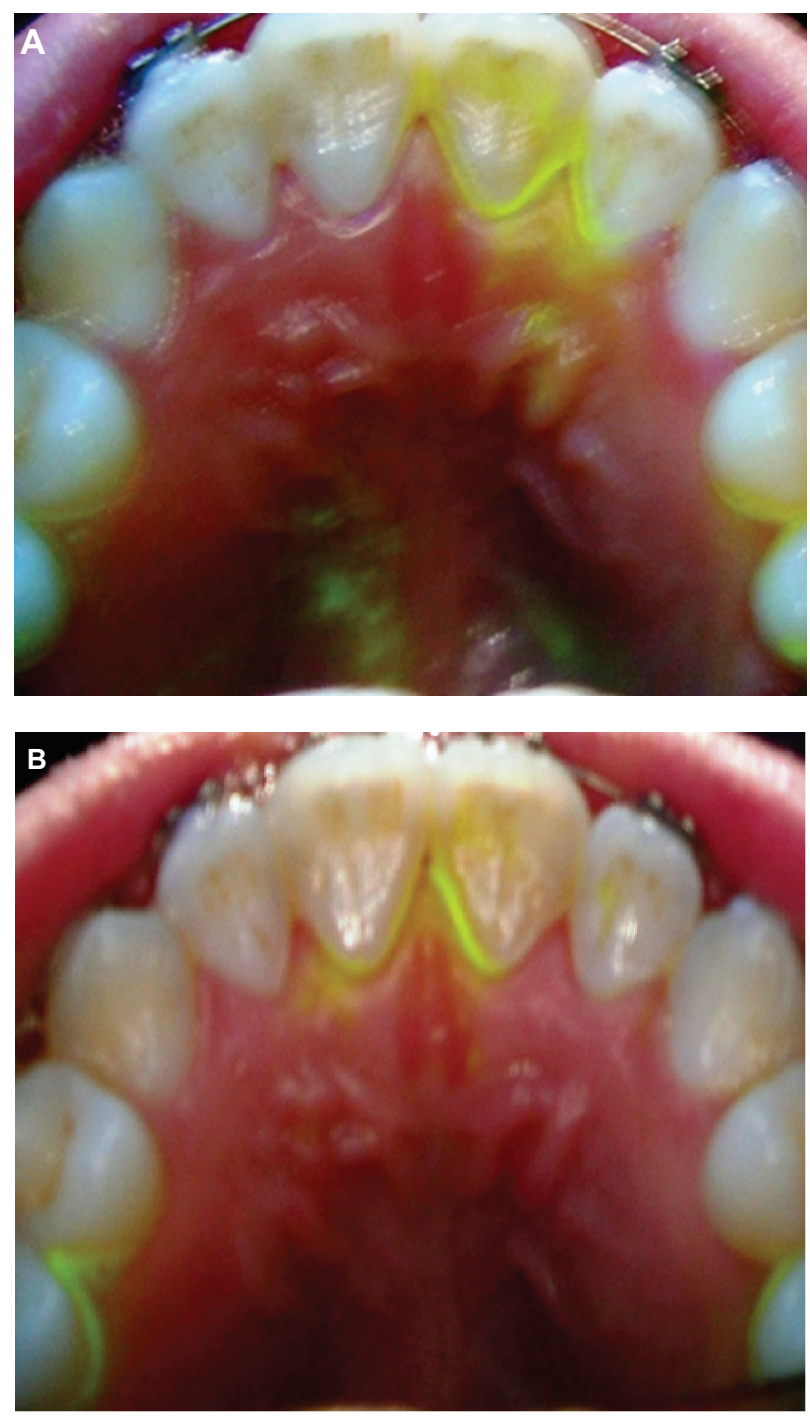

Figure 3 (A) Lateralization of the lingual apex and (B) repositioning of the lingual apex to the middle position.

of atypical deglutition was promisingly stable. The further improvement observed at three-month follow-up may be the result of a progressive normalization process, most likely related to synchronization of activity in the swallowing control areas of the brain.

\section{Disclosure}

Salvatore Rinaldi and Vania Fontani are the inventors of the radioelectric asymmetric conveyor.

\section{References}

1. Capozzi L, Negri PL. Atypical deglutition: clinico-therapeutic contribution. Ann Stomatol (Roma). 1966;15(4):249-270. Italian.

2. Casardi T, Ruju C, Melis MT. A method of phonetic re-education in cases of childhood atypical deglutition associated with orthodontic therapy. Dent Cadmos. 1984;52(Suppl 5):57-59. Italian.

3. Cohen FF, Vallado SY. Atypical deglutition. Rev Bras Odontol. 1978; 35(1):21-24. Portuguese. 
4. Farronato GP, Preteroti AM, Salvato A, Bruno E. Relation between skeletal open bite and atypical deglutition. Arch Stomatol (Napoli). 1982;23(1):53-74. Italian.

5. Jeanmonod A. Role of atypical deglutition in the development of masticatory apparatus dysfunction. Bull Acad Natl Chir Dent. 1988; 34(34):35-40. French.

6. Miotti F, Ferro R, Crapisi S. Inter-incisal spaces and atypical deglutition: considerations on reflexes and parafunctions. G Stomatol Ortognatodonzia. 1984;3(3):452-455. Italian.

7. Riccioli GA. Certain cases of atypical deglutition (the tongue is an important factor in the etiopathogenesis of many dento-maxillary abnormalities). Mondo Odontostomatol. 1972;14(3):461-465. Italian.

8. Santini RM, Collarini M, Taboada N, Tosi R, Schiavelli H, de Batkis T. The morphologic changes in atypical deglutition. Rev Asoc Odontol Argent. 1968;56(1):23-25. Spanish.

9. Tani G. Atypical deglutition as a cause of the incidence of interincisor diastema. (Preliminary note). Riv Ital Stomatol. 1965;20(10): 1258-1270. Italian.

10. Tenenbaum M, Berman GR. Atypical deglutition - myofunctional and mechanical therapy. Ortodoncia. 1971;35(69):30-45. Spanish.

11. Verkindere MT, Lodter C, Cadet D. Electromyographic studies of typical and atypical deglutition. Chir Dent Fr. 1984;54(264):51-55. France.

12. Monaco A, Cattaneo R, Spadaro A, Marchetti E, Barone A. Prevalence of atypical swallowing: a kinesiographic study. Eur J Paediatr Dent. 2006;7(4):187-191.

13. Garattini G, Crozzoli P, Grasso G. Etiopathogenesis and early treatment of malocclusions related to persistence of atypical deglutition. Mondo Ortod. 1991;16(2):149-156. Italian.

14. Machado AJ Jr, Crespo AN. Radiographic position of the hyoid bone in children with atypical deglutition. Eur J Orthod. February 4, 2011. [Epub ahead of print].

15. Sheppard IM, Sheppard SM. The relationship of vertical dimension of atypical swallowing with complete dentures. J Prosthet Dent. 1977; 38(3):249-253.

16. Stormer K, Pancherz H. Electromyography of the perioral and masticatory muscles in orthodontic patients with atypical swallowing. J Orofac Orthop. 1999;60(1):13-23.

17. Tosello DO, Vitti M, Berzin F. EMG activity of the orbicularis oris and mentalis muscles in children with malocclusion, incompetent lips and atypical swallowing. Part I. J Oral Rehabil. 1998;25(11):838-846.

18. Tosello DO, Vitti M, Berzin F. EMG activity of the orbicularis oris and mentalis muscles in children with malocclusion, incompetent lips and atypical swallowing. Part II. J Oral Rehabil. 1999;26(8):644-649.

19. Viti M, Mazza A, Malan R, Montaldo A. Introductory note on atypical deglutition. Parodontol Stomatol (Nuova). 1985;24(2):31-46. Italian.

20. Wilson EM, Green JR. Coordinative organization of lingual propulsion during the normal adult swallow. Dysphagia. 2006;21(4):226-236.

21. Ciavarella D, Mastrovincenzo M, Sabatucci A, Parziale V, Chimenti C. Effect of the enveloppe linguale nocturne on atypical swallowing: surface electromyography and computerised postural test evaluation. Eur J Paediatr Dent. 2010;11(3):141-145.
22. Giuca MR, Pasini M, Pagano A, Mummolo S, Vanni A. Longitudinal study on a rehabilitative model for correction of atypical swallowing. Eur J Paediatr Dent. 2008;9(4):170-174.

23. Levrini A. Atypical deglutition and functional myotherapy. Mondo Ortod. 1977;19(3):24-48. Italian.

24. Louis P. The dentist and the speech therapist. A team for diagnosis and treatment of atypical deglutition. Inf Dent. 1991;73(35):3045-3052. French.

25. Weinberg LA. An evaluation of stress in temporomandibular joint dysfunction-pain syndrome. J Prosthet Dent. 1977;38(2):192-207.

26. Hebling SR, Cortellazzi KL, Tagliaferro EP, et al. Relationship between malocclusion and behavioral, demographic and socioeconomic variables: a cross-sectional study of 5-year-olds. J Clin Pediatr Dent. 2008;33(1):75-79.

27. Rinaldi S, Fontani V, inventors, Rinaldi S, Fontani V, assignees. Radioelectric asymmetric conveyer for therapeutic use. US patent EP1301241 (B1). October 11, 2006.

28. Rinaldi S, Fontani V, inventors, Rinaldi S, Fontani V, assignees Radioelectric asymmetric conveyer for therapeutic use. US patent 7,333,8592001.

29. Leichsenring F. Randomized controlled versus naturalistic studies: a new research agenda. Bull Menninger Clin. 2004;68(2):137-151.

30. Fabaron JP, Nadau R. The Payne method of the use of black light in the current diagnosis of atypical deglutition. Orthod Fr. 1976;47(0): 157-161. French.

31. Sulsente AR. Ottimizzazione Neuro Posturale di un Gruppo di Sciatori d'elite [Neuropostural optimization in a group of elite skiers] [thesis]. Corso di Laurea in Scienze delle Attività Motorie e Sportive, Università degli Studi di Verona, Italy, Università degli Studi di Verona; 2008.

32. Castagna A, Rinaldi S, Fontani V, Aravagli L, Mannu P, Margotti ML. Does osteoarthritis of the knee also have a psychogenic component? Psycho-emotional treatment with a radio-electric device vs intraarticular injection of sodium hyaluronate: an open-label, naturalistic study. Acupunct Electrother Res. 2010;35(1-2):1-16.

33. Collodel G, Moretti E, Fontani V, et al. Effect of emotional stress on sperm quality. Indian J Med Res. 2008;128(3):254-261.

34. Mannu P, Rinaldi S, Fontani V, Castagna A, Lotti Margotti M. Radio electric treatment vs escitalopram in the treatment of panic disorders associated with major depression: an open-label, naturalistic study Acupunct Electrother Res. 2009;34:135-149.

35. Rinaldi S, Fontani V, Aravagli L, Margotti ML. Psychological and symptomatic stress-related disorders with radio-electric treatment: psychometric evaluation. Stress Health. 2009; Published Online: January 20, 2010.

36. Rinaldi S, Fontani V, Aravagli L, Mannu P. Psychometric evaluation of a radio electric auricular treatment for stress related disorders: a double-blinded, placebo-controlled controlled pilot study. Health Qual Life Outcomes. 2010;8(1):31.

37. Rinaldi S, Fontani V, Moretti E, et al. A new approach on stress-related depression and anxiety: neuro-psycho- physical-optimization with radio electric asymmetric-conveyer. Indian J Med Res. 2010;132: 189-194.
Journal of Multidisciplinary Healthcare

\section{Publish your work in this journal}

The Journal of Multidisciplinary Healthcare is an international, peerreviewed open-access journal that aims to represent and publish research in healthcare areas delivered by practitioners of different disciplines. This includes studies and reviews conducted by multidisciplinary teams as well as research which evaluates the results or conduct of such teams or

\section{Dovepress}

healthcare processes in general. The journal covers a wide range of areas and welcomes submission from practitioners at all levels, from all over the world. The manuscript management system is completely online and includes a very quick and fair peer-review system. Visit http://www.dovepress.com/testimonials.php to read real quotes from published authors. 